\title{
Cyclophosphamide/Vincristine/Dacarbazine Regimen
}

National Cancer Institute

\section{Source}

National Cancer Institute. Cyclophosphamide/Vincristine/Dacarbazine Regimen. NCI

Thesaurus. Code C156261.

A regimen consisting of cyclophosphamide, vincristine and dacarbazine that can be used for the treatment of malignant paragang lioma and pheochromocytoma. 